\title{
Ambulation training of neurological patients on the treadmill with a new Walking Assistance and Rehabilitation Device (WARD)
}

\author{
F Gazzani†, ${ }^{3}$, M Bernardi*,1,2, A Macaluso ${ }^{1}$, D Coratella ${ }^{1}$, JF Ditunno $\mathrm{Jr}^{4}$, V Castellano ${ }^{2}$, M Torre ${ }^{3}$, V Macellari ${ }^{3}$ \\ and M Marchetti ${ }^{1,2}$ \\ ${ }^{1}$ Istituto di Fisiologia Umana, Università 'La Sapienza', Rome, Italy; ${ }^{2}$ I.R.C.C.S. Ospedale di Riabilitazione, S. \\ Lucia, Rome, Italy; ${ }^{3}$ Lab. di Ingegneria Biomedica, Istituto Superiore di Sanità, Rome, Italy; ${ }^{4}$ Thomas Jefferson \\ University Hospital, Philadelphia, Pennsylvania, USA
}

\begin{abstract}
Study design: Patients with neurological walking impairment were rehabilitated with a new system, consisting of an apparatus to constantly relieve the body weight and a treadmill: The Walking Assistance and Rehabilitation Device (WARD). Patients were evaluated before and after rehabilitation with clinical scales and physiological measurements.

Objectives: To evaluate the effectiveness of the WARD in improving walking capability in these patients.

Setting: The study was carried out in a clinical environment (IRCCS S. Lucia, Rehabilitation Hospital, Rome, Italy).

Methods: Seven patients (six with spinal cord injuries, one with brain injury) underwent a $1-$ 2 month training period with the WARD. During the WARD training the body weight constant unloading (BWCU) applied to the patient through the WARD was regularly evaluated. Oxygen consumption, carbon dioxide production and heart rate were measured in order to obtain energy and cardiac costs of walking. These measurements were carried out while walking with the WARD at an appropriate treadmill speed (ATS) and in the open field at the most comfortable speed (MCS). All measurements, in addition to clinical scores related to the walking capability, were carried out at the beginning of the WARD training period (BWT) and at the end (EWT). At the EWT the patients were tested walking with the WARD observing the same BWT conditions (same ATS and BWCU), referred to as beginning conditions second measurements (BCSM). The relationships between physiological costs and ATS were described through second order polynomial regression curves and studied.

Results: Comparing the data obtained at the BWT and EWT, the following results were found significantly different: (1) Clinical scores improved; (2) All patients increased their ATS; (3) The BWCU was reduced; (4) The Walking Energy Cost (WEC) and the Walking Cardiac Cost (WCC) measured when walking with the WARD at the ATS improved in all patients; and (5) The WEC and WCC measured in the open field improved in all patients. The WEC vs ATS curve found at the EWT in the BCSM was found significantly different from the BWT curve, demonstrating a major improvement due to the WARD training.

Conclusion: Despite some limitations due to sample size and functional ambulation scale, this study has demonstrated that the WARD training is effective in improving the walking capability and efficiency of the patients.
\end{abstract}

Keywords: body weight support; treadmill; spinal cord injury; locomotion training

\section{Introduction}

One of the major purposes of effective rehabilitation is the restoration of independent ambulation as soon as possible. Many patients have to cope with neurological and orthopedic disorders which oblige them to spend prolonged periods in bed. This partial immobilization

*Correspondence: M Bernardi, Istituto di Fisiologia Umana, Facolta' di Medicina e Chirurgia-Universita' degli Studi di Roma 'La Sapienza', Piazzale Aldo Moro, 5-00185-Roma, Italy

$\dagger$ Fabio Gazzani died during the period of this study becomes a risk factor which worsens the initial impairment because it can cause further complications. Therefore, early mobilization of the patients is one of the prime goals in rehabilitation. However, it is difficult to train patients for ambulation if they are unable to support their own weight or stand erect because of neuromuscular weakness. This is due in part to the problems encountered with gravity and inertia forces when an individual attempts to assume the erect posture and move the legs forward. These problems in 
completely paralysed patients have been overcome by the use of orthoses (Reciprocating gait orthoses) and Functional Electrical Stimulation systems (defined as hybrid orthoses ${ }^{1}$ ). The hybrid orthoses, however, do not solve the problem of the high energy cost ${ }^{2}$ which has been demonstrated as being due to the high mechanical work performed and to the low efficiency of this kind of walking. ${ }^{3}$ More recently, trials of ambulation in less severely paralysed patients with body weight unloading (BWU) have also been shown to be effective. ${ }^{4-7}$

Since 1995 we have been successful in reducing the energy cost of ambulation in complete spinal cord injury patients by training them with Reciprocating Gait Orthoses on a treadmill. ${ }^{8-10}$ In this case the treadmill provided an external power supply to the patient thus reducing walking cost. Because of the quoted recent advances in rehabilitation with BWU and based on our own experience, we were encouraged to design a new support device to be utilized in treadmill training. ${ }^{11}$ This system, termed the Walking Assistance and Rehabilitation Device (WARD), includes both a BWU apparatus and a treadmill (Figure 1a). The system is able to exert a constant force against gravity during all the phases of the gait, in spite of the vertical swings of the body's centre of gravity (in other words, regardless of the position of the body's centre of gravity). In this study we investigated the efficacy of the use of the WARD in the rehabilitation of neurological patients with locomotor impairment. We hypothesized that the patient trained with this protocol would improve their clinical score and the cost indices of walking.

\section{Materials and methods}

Seven patients, six with spinal cord injuries and one with brain injury were selected from a rehabilitation centre in Rome (IRCCS S. Lucia). Their anthropometric data, specific diagnoses and time from injury to rehabilitation are listed in Table 1. According to Wernig et $a l^{12}$ six out of seven subjects were "chronic patients', entering the WARD program after a period ranging between 0.5 and 45 years from the beginning of pathology. All patients agreed to participate in the
WARD project and signed an informed consent form. They came from other rehabilitation centres where they had undergone traditional physiotherapy for a period ranging from 1-20 months. This training protocol consisted of a progressive rehabilitation program which included: passive or active (when possible) kinesis in bed; relaxation exercises; adaptation to standing posture using inclined bed (gradually verticalized); standing between parallel bars; wheelchair use training; walking between parallel bars, locomotion using walker; locomotion using tripod; locomotion using crutches. During the whole rehabilitation program the patients were supervised by a physiatrist and assisted by a physiotherapist through specific and individual treatment.

All patients were classified according to an ambulation scale, at the beginning of the traditional physiotherapy, that is at the beginning of the rehabilitation treatment (BRT), at the beginning of the WARD training (BWT) and at the end of the WARD training (EWT). The scale used was the Adapted Patient Evaluation Conference System (APECS), ${ }^{13}$ chosen because it utilized adaptive devices in the classification. The score which we refer to at BRT is based on the personal case sheet of the patient. Three out of seven patients did not show any improvement during traditional physiotherapy (time period from BRT to BWT). At the beginning of the WARD training four out of seven patients were unable to walk independently.

\section{The WARD description}

The WARD ${ }^{11}$ consists of a body weight unloading (BWU) apparatus, a suspension harness, a treadmill and control and data acquisition equipment (Figure 1a). Part of the BWU apparatus is placed over the treadmill (Enraf Nonius, Holland) where the patient walks. The treadmill walking surface is $0.6 \mathrm{~m}$ wide and $1.5 \mathrm{~m}$ long. The treadmill speed can be finely graded up to $0.1 \mathrm{~km} . \mathrm{h}^{-1}$ through a separate electronic digital system.

The BWU apparatus consists of a pneumatic system (to relieve the patient of part of the body weight) and a trolley which moves backwards and forwards (to

Table 1 Characteristics of subjects

\begin{tabular}{|c|c|c|c|c|c|c|}
\hline Subject & $\operatorname{Sex}$ & $\begin{array}{c}\text { Age } \\
\text { (years) }\end{array}$ & $\begin{array}{c}\text { Stature } \\
(m)\end{array}$ & $\begin{array}{c}\text { Mass } \\
(\mathrm{kg})\end{array}$ & Etiology & $\begin{array}{c}\text { Time from injury to the } \\
\text { beginning of WARD training } \\
\text { (BWT) (months) }\end{array}$ \\
\hline 1 & M & 45 & 1.70 & 76 & Trauma L1 & 12 \\
\hline 2 & M & 38 & 1.87 & 73 & Trauma C5-C6 & 6 \\
\hline 3 & M & 61 & 1.67 & 85 & Cranial trauma & 444 \\
\hline 4 & M & 70 & 1.70 & 75 & Tumour L5 & 3 \\
\hline 5 & $\mathrm{~F}$ & 43 & 1.56 & 56 & Congenital paraparesis & 540 \\
\hline 6 & $\mathrm{~F}$ & 62 & 1.65 & 56 & Degenerative paraparesis & 36 \\
\hline 7 & M & 18 & 1.80 & 65 & Trauma L1 & 7 \\
\hline
\end{tabular}


allow for the patient's different positions on the treadmill). The trolley runs along a steel beam track placed above the centre of the treadmill. This track is mounted onto the main steel tube frame of the WARD. The pneumatic system is composed of a pneumatic cylinder anchored under the trolley and a box containing the electric circuits, all the pneumatic components and the analog digital (A/D) converter. The system exerts the programmed upward force constantly, regardless of the position of the body or the body's centre of gravity. We therefore refer to this body weight support system as body weight constant unloading (BWCU). The pneumatic system is controlled through a personal computer and is able to support the patient beneath the arms at a desired percentage of his body weight. The upward force is

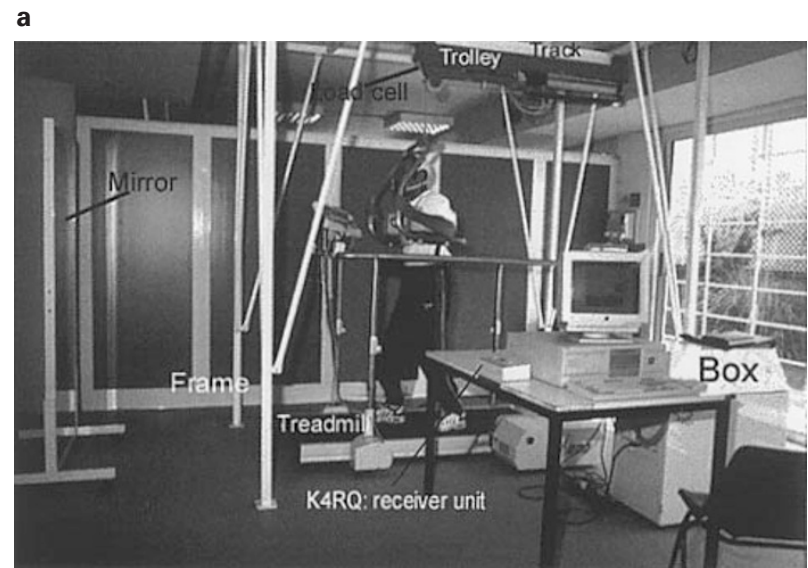

b

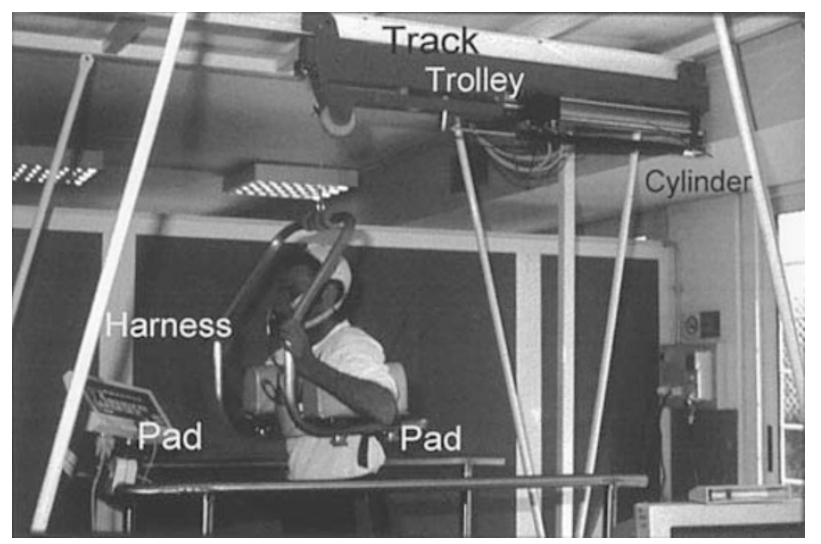

Figure 1 (a) The Walking Assistance and Rehabilitation Device (WARD) includes both a body weight unloading system and a treadmill. The system is able to exert a constant force against gravity during all the phases of the gait, in spite of the vertical swings of the body's centre of gravity. Each fundamental part of the system is indicated in the figure. The Box contains electric circuits, the pneumatic components and the analogic/digital converter. (b) Details of the WARD: a close up of the harness and pads applied to the patient through a harness consisting of a metal frame shaped in a way as to surround the patient's chest supporting the patient's armpits through adaptable pads (see Figure 1b, detail of Figure 1a).

\section{Training protocol}

The training period lasted approximately 1-2 months. During this period the hospital fees were paid mainly by the State through the National Health Service. This payment procedure does not allow a hospitalization period longer than 2 months. During the WARD treatment, all patients were involved in three treadmill training sessions per week, under the supervision of the physiotherapist: each training session lasted about $30 \mathrm{~min}$. At the beginning of the WARD training the patients were raised from a sitting to standing posture through the WARD. They spent $3 \mathrm{~min}$ in the standing posture on the treadmill. During this period the BWCU was adjusted for each patient at a constant value equal to $45 \%$ of their body weight. The subjects became familiar with the pull of the harness and tried to find a perfect balance. Then the WARD operator started the treadmill and progressively increased the speed up to a value which the physiotherapist considered the fastest speed which allowed regular and safe walking. The physiotherapist advised the patient in order to obtain the most fluent and symmetrical movement of the legs and at the same time considered patient feedback regarding comfort. We named the fastest speed which fulfils these criteria 'appropriate treadmill speed' (ATS). As the ATS was achieved, the BWCU could be progressively reduced to a value which however still guaranteed adequate support and balance of the patient. The body weight unloading assessment was also made according to the personal demand of the patient. Under these conditions the patient walked on the treadmill as long as possible (up to a maximum of $20 \mathrm{~min}$ ).

At the beginning of each new training session the previous value of ATS and BWCU was selected as the initial training condition. After $3 \mathrm{~min}$ of familiarization and considering patient feedback, the same procedure as described earlier was adopted in order to increase the ATS and to reduce the BWCU. In summary, the treadmill was always adjusted to the ATS and the BWCU was adjusted to a level which provided adequate support and balance of the patient (ie the BWCU was reduced to the minimal value which maintained the subject's balance without any risk). As already specified, according to the rules of the Italian Health Service, the financial support from the State expires after 2 months. The WARD training lasted in accordance with this financial support. The WARD training period was started as soon as possible after hospital admission when permitted by the general condition of the patient. For each patient the duration of training is given in Table 2 . 


\section{Gait evaluation}

In order to test the improvement obtained with the WARD training, the following measurements were taken at the beginning (BWT) and end (EWT) of the training period: (1) Speed when walking with the WARD, ie the treadmill speed that the physiotherapist selected as ATS; (2) BWCU during walking with the WARD, expressed as an absolute value (Newtons) and as a percentage of the body weight of the patient; (3) Number of double steps per minute the subject performed when walking with the WARD and, by calculation, length of double step (metres); (4) Energy and cardiac costs when walking with the WARD at the ATS; and (5) Energy and cardiac costs when walking in the open field at the most comfortable speed (MCS), ie, the speed which the subject selected as the most comfortable, aware that it was to be sustained for at least $5 \mathrm{~min}$. These measurements were taken from four subjects. A gym for physiotherapy constituted the open field. A $25 \mathrm{~m}$ long circular pathway was outlined. At every $5 \mathrm{~m}$ a reference point was made clearly visible to allow the patient's walking speed to be properly measured.

Additional measurements were taken when the costs were assessed at the EWT and the subjects were asked to walk with the WARD again under exactly the same conditions as BWT (same ATS and BWCU). This was done to evaluate possible improvement in walking efficiency. We referred to this evaluation as 'beginning conditions second measurements' (BCSM).

As in previous papers on the assessment of walking impairment, ${ }^{14,15}$ we considered two costs as indices of walking: the walking energy cost (WEC) and the

Table 2 Clinical score of each patient (Adapted Patient Evaluation Conference System (APECS); Korner-Bitensky et $a l^{13}$ ) at the beginning of rehabilitation treatment (BRT), at the beginning of WARD training (BWT) and at the end of WARD training (EWT)

\begin{tabular}{lcccccc}
\hline Subject & $B R T$ & Score & BWT & Score & EWT & Score \\
\hline 1 & $07 / 03 / 95$ & 2 & $11 / 03 / 96$ & 2 & $18 / 04 / 96$ & 3 \\
2 & $23 / 09 / 95$ & 1 & $16 / 10 / 95$ & 1 & $10 / 12 / 95$ & 3 \\
3 & $05 / 07 / 94$ & 1 & $11 / 03 / 96$ & 2 & $07 / 04 / 96$ & 3 \\
4 & $24 / 01 / 96$ & 0 & $11 / 03 / 96$ & 1 & $18 / 04 / 96$ & 2 \\
5 & $18 / 10 / 95$ & 3 & $28 / 11 / 95$ & 3 & $20 / 01 / 96$ & 6 \\
6 & $06 / 09 / 95$ & 3 & $09 / 10 / 95$ & 4 & $20 / 11 / 95$ & 4 \\
7 & $10 / 05 / 96$ & 5 & $24 / 11 / 96$ & 6 & $24 / 12 / 96$ & 7 \\
\hline
\end{tabular}

0 , Not assessed; 1, Needs maximal assistance from two people or an assistive device +1 person; 2 , Requires minimal assistance from another person with or without an assistive device; 3, Requires supervision and an assistive device; 4, Requires supervision for safety; no assistive device needed; 5, Independent but cannot walk at a reasonable rate and/or has poor endurance (ie $10 \mathrm{~m}$ or less with or without an assistive device). Difficulty ambulating outdoors; 6, Independent with assistive device. No supervision required. Person can ambulate indoors and outdoors under simulated conditions, ie, ramp, carpet, curb, uneven surface, any season; 7, Within normal limits - functionally independent walking cardiac cost (WCC). At the BWT, they were assessed, on the same day, 2 days after the measurements of points 1, 2 and 3. Two days after the EWT, all final measurements (points 1, 2, 3, 4, 5 and BCSM) were taken. In both testing sessions (BWT and EWT), whether the patients started with the measurements of point 4 , point 5 or BCSM was random, to avoid any systematic error. Between taking the three measurements, a rest period of at least $30 \mathrm{~min}$ was respected, to exclude any fatigue effect. All tests were performed in the morning and at least $2 \mathrm{~h}$ after breakfast.

In order to assess the walking costs, we measured oxygen consumption $\left(\mathrm{VO}_{2}\right)$, carbon dioxide production $\left(\mathrm{VCO}_{2}\right)$, pulmonary ventilation (VE) and heart rate (HR) by means of telemetric apparatus (K4RQ, Cosmed, Italy). The apparatus consists of a portable unit, a portable mask, a Polar Sport tester ${ }^{\mathrm{TM}}$ electrode couple and a receiving unit (Figure 2). The portable unit, which weighs $600 \mathrm{~g}$, includes a thermostated galvanic fuel cell oxygen analyzer, a thermostated infrared non dispersive carbon dioxide analyzer, an adapted heart rate monitor (Polar Electro Oy, Finland) and the device for the teletransmission. The mask includes a $28 \mathrm{~mm}$ diameter bi-directional digital turbine used as a flowmeter. A capillary tube is used to collect samples of the expired air to be analyzed in the portable unit. According to our protocol, ${ }^{14,15}$ after at least $3 \mathrm{~min}$ in which the patients, at rest and in standing posture, had familiarized themselves with the K4RQ apparatus, they were requested to walk. We started recording with the K4RQ apparatus straight after the familiarization procedure in order to obtain a

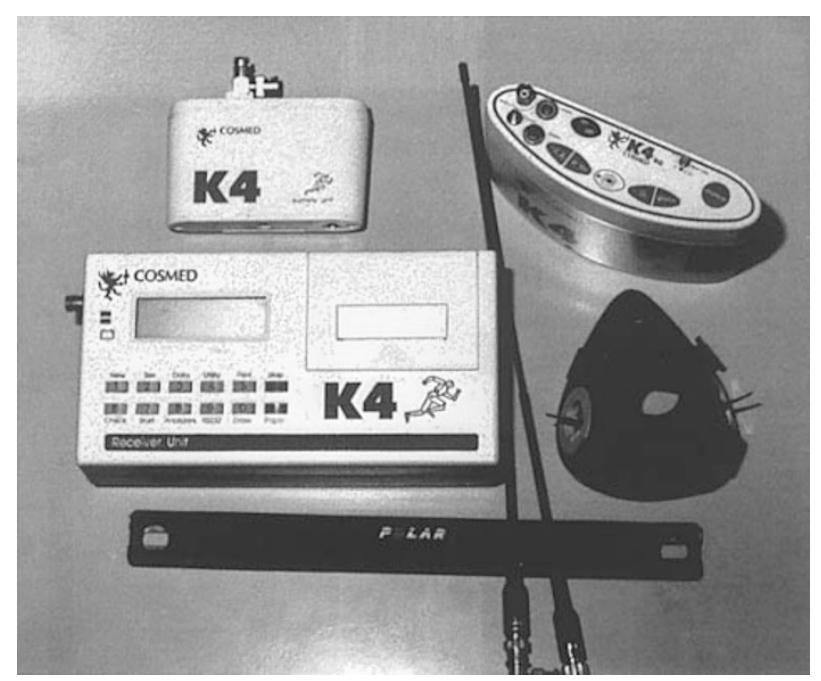

Figure 2 K4RQ, Cosmed: telemetric device for the metabolic and cardiac measurements. In a clockwise direction, starting from the top left: battery, portable unit, mask, electrodes for heart rate measurement, antennas and receiver unit 
measurement at rest. Each subject was requested to walk for at least $3 \mathrm{~min}$ in order to reach steady state conditions, as represented by stable $\mathrm{VO}_{2}, \mathrm{VCO}_{2}$ and HR values. The values were collected every $30 \mathrm{~s}$ and those collected in the fourth and fifth minutes were used to assess a mean value to be used in this research.

To calculate the costs, following Zamparo et al ${ }^{16}$ and according to Bernardi et $a l^{15}$ we used the overall measurements, ie the absolute value without subtracting the rest value (basal value). The overall WEC was calculated as the amount of oxygen consumed per kilogram $(\mathrm{kg})$ of body mass and per unit distance (expressed in $\mathrm{J} \cdot \mathrm{kg}^{-1} \cdot \mathrm{m}^{-1}$ ). The following formula was used: $\mathrm{WEC}=\mathrm{E}_{\mathrm{w}} / \mathrm{S}$; where $\mathrm{E}_{\mathrm{w}}$ is the energy intake during walking in $\mathrm{J}_{\mathrm{kg}} \mathrm{kg}^{-1} \cdot \mathrm{min}^{-1}$ and $\mathrm{S}$ is the speed in m. $\min ^{-1}$.

The overall walking cardiac cost (WCC) was calculated as the number of heart beats per unit distance (beats. $\mathrm{m}^{-1}$ ) according to the formula: $\mathrm{WCC}=\mathrm{HR}_{\mathrm{w}} / \mathrm{S}$. In this case $\mathrm{HR}_{\mathrm{w}}$ is the HR during walking in beats. $\min ^{-1}$ and $\mathrm{S}$ is the speed in $\mathrm{m} \cdot \mathrm{min}^{-1}$. WEC and WCC will be called Physiological Costs. This term will be used when the same concept applies to both of them.

\section{Statistical analysis}

To compare the results obtained before and after the WARD training, Student's $t$-tests for paired data were used to evaluate the difference in: clinical scores, ATS, number and length of double steps when walking with the WARD, BWCU, WEC and WCC when walking with the WARD, WEC and WCC when walking in the open field. The Student's $t$-test for paired data was also used to compare the WEC and WCC when walking with the WARD at the BCSM with the respective physiological costs of the BWT measurements. Second order polynomial regression curves were used to fit the WEC vs ATS data collected from the patients when walking with the WARD before and after the WARD training. In this way we obtained three curves: one curve for BWT data and two curves for the EWT data (point 5 and BCSM). The regression curves were checked for statistical significance and compared to test any resulting differences between them. To accomplish the latter the curves were made linear and the intercepts and coefficients of regression were compared.

Possible differences in the physical fitness of the patients after the WARD training were checked. To achieve this aim the relationships between WEC and WCC were described fitting the BWT and BCSM data through two first order polynomial regression curves. The BWT and BCSM curves so obtained were statistically compared.

All the statistical evaluations were carried out according to Sachs. ${ }^{17}$ The level of significance was set at $P<0.05$.

\section{Results}

The comparison between the measurements before and after the WARD training period demonstrated that an impressive improvement was achieved by all patients. Our results, expressed as a comparison using the Student's $t$-test for paired data (BWT data vs EWT data), can be schematically reported as follows: (1) Clinical scores, based on the APECS scale, ${ }^{13}$ showed a statistically significant difference after the WARD training. Indeed, all patients but one improved their scores. Table 2 shows the APECS scale, with the scores of each patient at the beginning of traditional physiotherapy (Beginning of Rehabilitation TherapyBRT), at the beginning of the WARD training (BWT) and at the end of the WARD training (EWT). It is noteworthy that three out of seven patients can be considered as having been stationary when the WARD training started, ie, their scores remained the same throughout the whole traditional rehabilitation period. Even more impressive is the fact that two patients, who were completely wheelchair bound at the BWT (score 1), increased their walking capability to score 3. (2) The patients' improvement when walking on the treadmill is demonstrated by the statistically significant improvement in ATS. In fact all subjects increased

Table 3 Appropriate Treadmill Speed (ATS; metres per minute). Double Step Length (DSL; metres), Double Step Rate (DSR; DS per minute), Body Weight Constant Unloading (BWCU; Newtons): absolute, mean and standard deviation values at the beginning of the WARD training (BWT), and the end of the WARD training (EWT)

\begin{tabular}{|c|c|c|c|c|c|c|c|c|}
\hline \multirow[b]{2}{*}{ Subjects } & \multicolumn{2}{|c|}{$A T S\left(\mathrm{~m} . \mathrm{min}^{-1}\right)$} & \multicolumn{2}{|c|}{$D S L(\mathrm{~m})$} & \multicolumn{2}{|c|}{$D S R\left(\right.$ DS. $\left.\min ^{-1}\right)$} & \multicolumn{2}{|c|}{$B W C U(\mathrm{~N})$} \\
\hline & $B W T$ & $E W T$ & $B W T$ & $E W T$ & $B W T$ & $E W T$ & $B W T$ & $E W T$ \\
\hline 1 & 25 & 33.3 & 0.83 & 0.83 & 30.1 & 40 & 221 & 182 \\
\hline 2 & 8.3 & 16.6 & 0.71 & 0.83 & 11.7 & 20 & 442 & 288 \\
\hline 3 & 13.3 & 25 & 0.48 & 0.79 & 27.8 & 32 & 202 & 202 \\
\hline 4 & 6.6 & 23.3 & 0.59 & 0.83 & 11.2 & 28 & 326 & 326 \\
\hline 5 & 16.6 & 41.6 & 0.52 & 1 & 32 & 42 & 211 & 106 \\
\hline 6 & 20 & 25 & 0.69 & 0.74 & 29 & 34 & 211 & 106 \\
\hline 7 & 16.6 & 41.6 & 0.67 & 1.05 & 25 & 40 & 250 & 182 \\
\hline Mean & 15.2 & 29.4 & 0.64 & 0.88 & 24 & 33.5 & 266 & 198 \\
\hline SD & 5.95 & 8.88 & 0.11 & 0.11 & 8.7 & 7.8 & 81.9 & 78 \\
\hline
\end{tabular}


their ATS after the WARD training (Table 3). The mean ATS increased from $15.2+5.9$ to $29.4 \pm 8.9 \mathrm{~m} \cdot \mathrm{min}^{-1}$. This improvement in speed is due to a statistically significant increase in both the rate and the length of the double steps, as also shown in Table 3. The mean number of double steps per minute (double step rate-DSR) increased from $24 \pm 8.7$ at the BWT to $33.5 \pm 7.8$ at the EWT. The average length of the steps increased from $0.64 \pm 0.11$ metres at BWT to $0.87 \pm 0.11$ metres at EWT. (3) In five out of seven patients it was possible to reduce the BWCU throughout the WARD training (see Table 3). The reduction in BWCU after the WARD training was statistically significant. The BWCU decreased from a mean value of $266 \pm 82 \mathrm{~N}$ to a mean value of $198+78$. The BWCU, expressed as a percentage of the body weight which was unloaded, decreased from a mean value of $39 \pm 12 \%$ to $29 \pm 11 \%$. (4) Both the physiological costs (WEC and WCC) measured at the ATS decreased in all patients as a result of the WARD training. The mean WEC decreased from $16.2 \pm 6.6$ to $8.4 \pm 1.4$. The mean WCC decreased from $8.41 \pm 3.7$ to $4.3 \pm 1.2$ beats. $\mathrm{m}^{-1}$. The WEC and WCC data, with the mean values and the standard deviations, measured at the BWT and at the EWT are shown in Table 4. The Table also shows the second measurements taken at the EWT observing the same conditions as at BWT (BCSM). Both the Physiological Costs showed a statistically significant decrease at the BCSM. The WEC mean value decreased to $12.9 \pm 5.5 \mathrm{~J} \cdot \mathrm{kg}^{-1} . \mathrm{m}^{-1}$. The WCC mean value decreased to $7.6 \pm 3.2$ beats. $\mathrm{m}^{-1}$.

The graph of Figure 3 shows the WEC values of each subject measured at the BWT and at the EWT plotted as a function of the appropriate treadmill speed (ATS). Each subject is represented with a different symbol. Because each patient increased the ATS and decreased the WEC, each EWT point on the graph has moved rightwards and downwards with respect to the corresponding BWT point. The BCSM WEC data constitute a third set of points shown in the graph. These data, which were taken at the same treadmill speed as at BWT, have moved downwards with respect to the corresponding BWT data. Each set of data was fitted by a second order regression curve. The following equations and coefficients of determination were found:

$$
\begin{aligned}
\text { BWT WEC }= & 41.7 \quad 2.59 \mathrm{ATS}+0.052 \mathrm{ATS}^{2} \\
& \left(r^{2}=0.995\right) ; \\
\text { EWT WEC }= & 23.6 \quad 1.03 \mathrm{ATS}+0.016 \mathrm{ATS}^{2} \\
& \left(r^{2}=0.766\right) ; \\
\text { BCSM WEC }= & 32.06 \quad 1.86 \mathrm{ATS}+0.034 \mathrm{ATS}^{2} \\
& \left(r^{2}=0.925\right) .
\end{aligned}
$$

In each case the curves demonstrated a statistical significance. The three curves were compared from a statistical point of view after having used a procedure to make them linear. The intercepts and the slopes so obtained were compared. The BCSM and the EWT curves resulted statistically different from the BWT curve.

In order to have an estimation of the patients' physical fitness before and after the WARD training, the relationships between the WEC and the WCC were studied. The first order polynomial regression curves obtained fitting the data at the BWT and in the BCSM were compared. The data, the linear regression curves and the 95\% confidence intervals related to the two time periods are shown in Figure 4. The figure also includes the equations and the coefficients of determination of the two curves. No statistical difference was found between the two curves.

Three subjects performed a walking test in the open field at the BWT and at the EWT. In each subject, both the most comfortable speed (MCS) and the physiological costs improved. Open field measure-

\begin{tabular}{|c|c|c|c|c|c|c|}
\hline \multirow[b]{2}{*}{ Subjects } & \multicolumn{3}{|c|}{$W E C\left(\mathrm{~J} \cdot \mathrm{kg}^{-1} \cdot \mathrm{m}^{-1}\right)$} & \multicolumn{3}{|c|}{$W C C$ (beats. $\mathrm{m}^{-1}$ ) } \\
\hline & $B W T$ & $B C S M$ & $E W T$ & $B W T$ & $B C S M$ & $E W T$ \\
\hline 1 & 9.57 & 6 & 6.8 & 3.8 & 3.4 & 3 \\
\hline 2 & 23.6 & 18.4 & 10.9 & 12.9 & 11.8 & 6.5 \\
\hline 3 & 17.1 & 11.1 & 7.1 & 8.3 & 7 & 3.9 \\
\hline 4 & 26.8 & 22.3 & 7.9 & 14.1 & 12.2 & 4.1 \\
\hline 5 & 12.2 & 10.3 & 7.9 & 7 & 6.6 & 3.4 \\
\hline 6 & 11 & 10.9 & 9.1 & 6.3 & 6.7 & 5.5 \\
\hline 7 & 13.2 & 11.4 & 8.8 & 6.5 & 5.81 & 4 \\
\hline Mean & 16.21 & 12.9 & 8.35 & 8.4 & 7.6 & 4.34 \\
\hline SD & 6.6 & 5.5 & 1.3 & 3.7 & 3.2 & 1.2 \\
\hline
\end{tabular}
ments at the EWT were also taken in one subject

Table 4 Walking energy cost (WEC) and walking cardiac cost (WCC) measured while walking on the treadmill with the WARD at the beginning of the WARD training (BWT), at the end of the WARD training (EWT) and again at the end of the WARD training but under the same conditions (same appropriate treadmill speed and same body weight constant unloading) as before the WARD training (beginning conditions second measurements (BCSM)) 


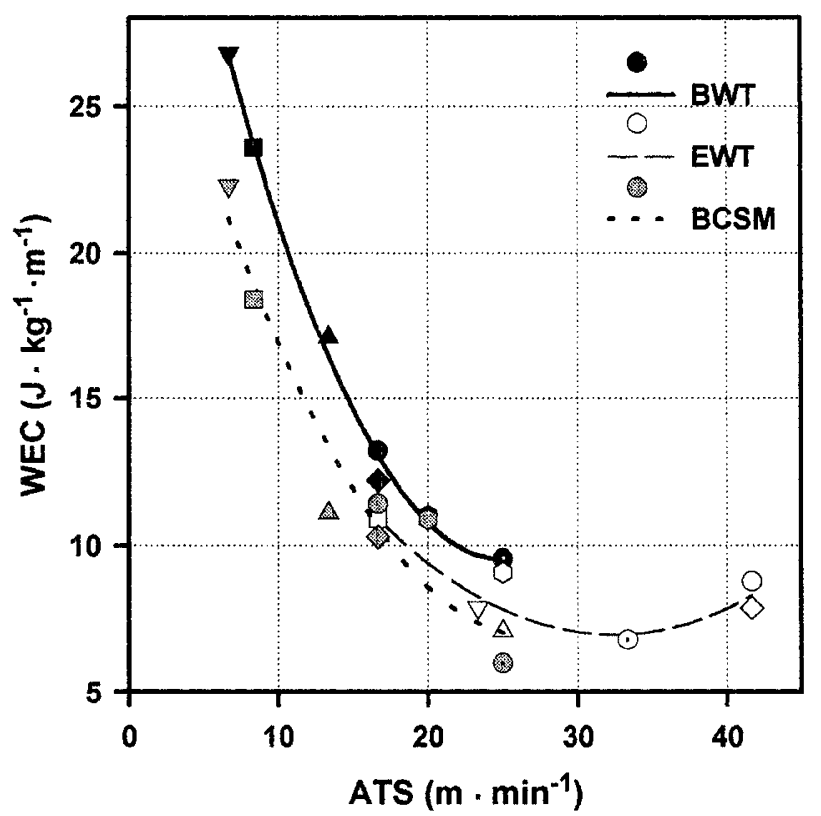

Figure 3 The graph shows the Walking Energy Cost values of each subject measured at the beginning of the WARD training (BWT) and at the end of the WARD training (EWT) plotted as a function of the appropriate treadmill speed (ATS). Each subject is represented with a different symbol. A third set of points represents the walking energy cost (WEC) data measured at the end of the WARD training (EWT) with the same conditions as at the BWT (BCSM). The three sets of data were fitted by second order regression curves. The equations and coefficients of determination found are shown in the text

who was unable to walk at the BWT. Table 5 shows the MCS, WEC and WCC data for these four subjects.

\section{Discussion}

The results of the study support our hypothesis because, after 1-2 months of the WARD training, all patients were able to walk on the treadmill at a reduced percentage of body weight constant unloading (BWCU), at a faster speed and with lower physiological costs. All patients but one improved their score on the ambulation scale. Of more practical importance, those patients measured in open field walking also showed a carryover improvement in walking speed and lowered their physiological costs after the training.

Two subjects at the beginning of the WARD training were unable to walk for more than a few steps and, to do this, each needed maximal assistance from two people. Using the WARD, these two patients were able to stand and walk unassisted from the first training session with the physiotherapist supervising only. At the end of the WARD training, both patients improved their walking capability up to a level which allowed them to walk in the 'open field' for at least $5 \mathrm{~min}$. This result can be considered a

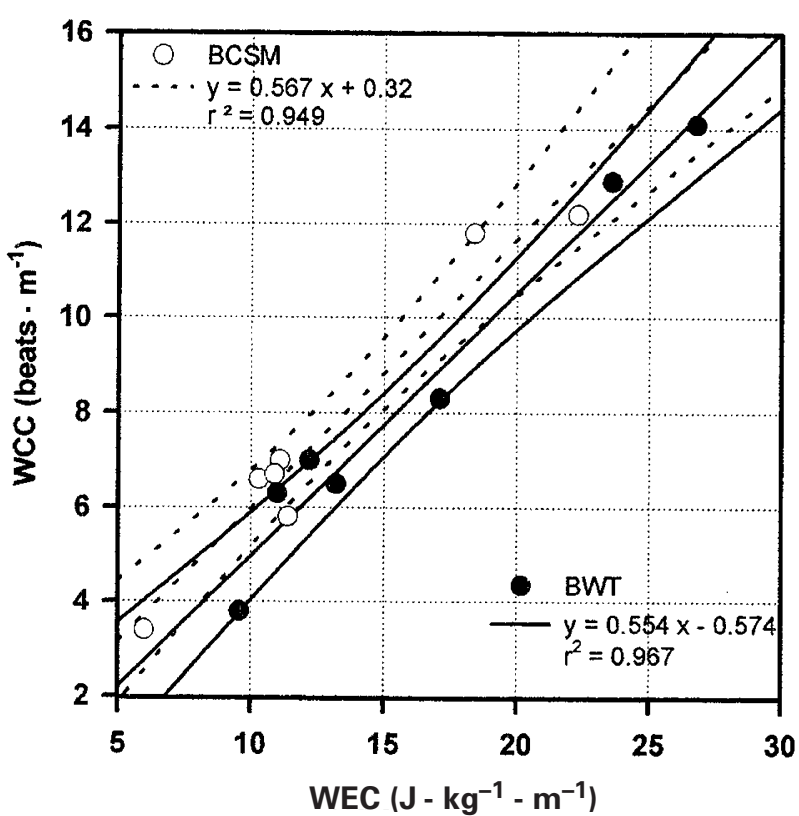

Figure 4 The filled circles are related to the relationship between the walking energy cost (WEC) and the walking cardiac cost (WCC) at the BWT. The unbroken lines represent the first order polynomial regression and the $95 \%$ confidence interval curves obtained fitting the data at the BWT. The unfilled circles are related to the relationship between the WEC and the WCC found at the EWT in the BCSM. The dotted lines represent the first order polynomial regression and the 95\% confidence interval curves obtained fitting the data in the BCSM. The equations and the coefficients of determination of the two curves are also represented

Table 5 Most comfortable speed (MCS), walking energy cost (WEC) and walking cardiac cost (WCC) measured while walking in the open field at the beginning of the WARD training (BWT) and at the end of the WARD training (EWT) (data on four patients). $\mathrm{U}=$ patient unable to walk

\begin{tabular}{lcccccc}
\hline & \multicolumn{2}{c}{$M C S$} & \multicolumn{2}{c}{$W E C$} & \multicolumn{2}{c}{$W C C$} \\
Subject & $B W T$ & $E W T$ & $B W T$ & $E W T$ & $B W T$ & $E W T$ \\
\hline 1 & 36 & 50 & 8.35 & 5.48 & 2.79 & 2.12 \\
3 & 33.9 & 40.5 & 9.22 & 6.51 & 3.42 & 2.7 \\
4 & $\mathrm{U}$ & 30 & $\mathrm{U}$ & 8.93 & $\mathrm{U}$ & 3.06 \\
7 & 35 & 41 & 10.2 & 8.8 & 3.6 & 2.9 \\
\hline
\end{tabular}

carryover effect of the WARD training. Our results are similar to those of other authors, ${ }^{5,7}$ who have demonstrated that the treatment with treadmill and body weight support was effective in the re-acquisition of walking ability in incomplete spinal cord lesion patients. These $e^{5,7}$ and other studies ${ }^{6,12,18}$ on patients either with complete and incomplete paraplegia or hemiplegia have demonstrated that below lesion spinal cord centres can 'learn' to use lower limb afferents to generate a locomotor pattern, and that the treadmill 
and the body support training method are effective in activating the spinal circuits.

Besides the re-acquisition of a neural motor program, the WARD training could promote the deambulation capability of paraplegic patients with other mechanisms. Our results demonstrate that the patients' walking improvement incorporated an increase in gait efficiency revealed by the reduction in the physiological costs. This fact is statistically supported by the Student's $t$-test and is clearly illustrated by the BWT and EWT curves shown in the graph of Figure 3. Indeed each subject increased his ATS and decreased his energy cost (WEC). In five out of seven patients the BWT WEC and the EWT WEC were measured at a lower body weight constant unloading (BWCU), and this fact certainly affected the results. We must consider that (a) the two subjects who were tested at the same BWCU showed the most evident reduction in cost, and (b) a hypothetical correlation factor, to compare WEC when lower BWCUs were applied, would show a further reduction in the cost of the remaining five subjects.

The improved efficiency is confirmed by the data of the three subjects who performed the walking test in the open field both BWT and EWT. In this case, there was no BWCU, but still the physiological costs of walking were lower and the speeds (speeds spontaneously chosen by the patients, MCS) higher at the EWT than BWT. Even more remarkable, in terms of improved walking efficiency, is the fact that at the EWT it was possible to measure the physiological costs in one patient who, at the BWT, was unable to walk long enough to take these measurements.

Up to a certain speed, walking efficiency improves when speed increases and this fact poses a problem in interpreting the data. Indeed, in healthy subjects the well known relationship between energy cost and speed is illustrated by a U-shaped curve with a minimum at $4-5 \mathrm{~km} \cdot \mathrm{h}^{-1}$ (economy or natural speed) and a progressively increasing cost at higher and lower speeds. ${ }^{19,20}$ At the low speed typical of a patient, even a normal subject shows a great deambulation disadvantage which has been demonstrated by energy data. ${ }^{3}$ A U-shaped curve was also obtained in walking impaired subjects. ${ }^{14}$ Thus the efficiency improvement we observed can be partially attributed to the increase in walking speed. The data we obtained at the end of the training when the patients walked with the WARD again at the same speed and with the same body support as before training (BCSM) resolves the problem. The comparison between the BWT WEC curve and BCSM WEC curve, shown in the graph of Figure 3, clearly indicates that the same locomotor act resulted to be less demanding in terms of muscle work. This suggests that some useless muscular contractions were reduced. The improved efficiency could be due to a reduction in the following: (a) agonist-antagonist cocontractions; (b) limb and trunk spasticity; and (c) unnecessary movements. Furthermore we can envisage a more efficient variation of the mechanical energy associated with the body segments (potential in kinetic energy and vice versa). In favour of our hypothesis are the works of Visintin and Barbeau, ${ }^{21}$ Dietz et $a l^{7}$ and Wernig and Muller. ${ }^{5}$ They have demonstrated through EMG and kinematic recordings that treadmill and body unloading training induces a reduction in gait asymmetry and in muscular spasticity as well as inducing the acquisition of a motor pattern more similar to the normal gait. It is noteworthy that the increase in treadmill walking speed was obtained in our patients with both an increase in rate and in length of the stride. The latter is in agreement with the hypothesis of a smoother and effortless swing, ie, fewer energy dispersing movements.

At the EWT and in the BCSM the subjects demonstrated lower WCC than at the BWT. This is in agreement with what we expected because of the reduced muscular work (and thus the reduced WEC) after training. However the difference between the BWT WEC and the BCSM WEC is in percentage equal to $20.1 \%$, while the difference between BWT WCC and BCSM WCC is $9.5 \%$. The reason that the reduction in WEC was bigger than the reduction in WCC could be due to the isometric effort during walking with the WARD. Indeed, the effort of maintaining the erect posture while walking on the treadmill at low speed entails isometric contractions of the upper limb muscles. Patients who have the benefit of partial body support must contract the upper limb muscles anyway in order to sustain the weight on the harness. The isometric effort can produce an increase in heart rate which is correlated to the force requested of the upper limb musculature and not to the total energy turnover. On the other hand with the increase in speed the WCC progressively dropped, as shown in the EWT data. Possibly at this level of workload the increase in aerobic exercise dominated over the heart activity with respect to the isometric effort.

Figure 4 shows the relationship between the metabolic energy demand during walking on the treadmill with the corresponding cardiac adjustments. The comparison of the BWT and BCSM curves, therefore, provides information about possible changes in cardiac fitness. Indeed, the measurements were taken at a similar workload because the subjects were requested to walk as they did at the BWT. It is evident from the comparison of the two linear regression curves (BWT and BCSM) that the subjects did not show any improvement in cardiac fitness, while they improved their walking efficiency (as illustrated in Figure 3). These results were actually expected because the WARD training did not have this specific goal, but was aimed at increasing the locomotor pattern of the subjects.

As already specified, most patients (six out of seven) showed an improvement in clinical scores of functional ambulation from one to three levels. This suggests that patients who show improvement in walking speed and reduced energy cost of this magnitude will also show 
functional ambulation improvement. The relationship among these measurements can be further studied to give more precise evaluations of the walking impairment of a patient. It is important to note that this improvement was obtained in a very short period of time compared with the period of previous rehabilitation.

There are, however, some limitations to this first report. First, a larger number of subjects are required in whom alternative therapies are compared in order to conclude that this method of training is superior to other methods. Second, the functional ambulation scale or APECS ${ }^{13}$ used in this study lacked two important features: (1) It does not include a description of the assistive device used, that is, it does not differentiate between a walker, one or two canes/crutches or a brace; and (2) The scale was validated for hemiplegia and not spinal cord injury. A functional ambulation scale for spinal cord injury needs to be developed similar to the one used in animal trials. $^{22}$ The scale in the animals studies has 21 levels as compared to the APECS with only seven levels and is likely to be more precise, and also highly reliable.

This new body weight system, which has the advantage of constant upward load, supports the body under the arms. Prolonged training sessions greater than $20 \mathrm{~min}$ may result in compression of neurovascular structures under the arms, besides inducing arm fatigue. The harness may need to be modified using a trunk support. ${ }^{23}$ Ide et $a l^{24}$ reported the use of a robotic device to support patients under the arms and trunk with a load-cell sensor which controlled shifts of the centre of gravity. Although similar to our device in regard to constant force, it was utilized on a circular pathway rather than on a treadmill. We suggest, however, that exercise on a treadmill offers the following advantages: (a) the speed is pre set by the physiotherapist; (b) the speed is constant during the exercise; and (c) the walking pattern is more easily controlled by the physiotherapist.

\section{References}

1 Solomonow M, et al. Reciprocating gait orthosis powered with electrical muscle stimulation (RGO II). Part I: Performance evaluation of 70 paraplegic patients. Orthopedics 1997; 20: $315-$ 324.

2 Nene AV, Hermens HJ, Zilvold G. Paraplegic locomotion: a review. Spinal Cord 1996; 34: 507-524.

3 Bernardi M, et al. The efficiency of walking in RGO paraplegic patients using a reciprocating gait orthosis. Paraplegia 1995; 33: $409-415$.
4 Finch L, Barbeau H, Arsenault B. Influence of body weight support on normal human gait: development of a gait retraining strategy. Phys Ther 1991; 71: $842-855$.

5 Wernig A, Muller S. Laufband locomotion with body weight support improved walking in persons with severe spinal cord injuries. Paraplegia 1992; 30: 229-238.

6 Hesse S, et al. Restoration of gait in nonambulatory hemiparetic patients by treadmill training with partial body-weight support. Arch Phys Med Rehabil 1994; 75: 1087-1093.

7 Dietz V, Colombo G, Jensen L, Baumgartner L. Locomotor capacity of spinal cord in paraplegic patients. Ann Neurol 1995; 37: $574-582$

8 Bernardi $\mathrm{M}$, et al. Use of treadmill in paraplegic rehabilitation. Proceeding of the 5th European Congress on Research in Rehabilitation, Helsinki, Finland, 28 May-1 June 1995. Journal of Rehabilitation Sciences 1995; 8: 48.

9 Bernardi M, et al. External power supply to improve RGO training. 35th Annual Scientific Meeting of the International Medical Society of Paraplegia, Atlanta, Georgia, USA, August $18-20,1996$. Book of Abstracts.

10 Felici F, Bernardi M, Rodio A, Marchettoni P, Castellano V, Macaluso A. Rehabilitation of walking for paraplegic patients by means of a treadmill. Spinal Cord 1997; 35: $383-385$.

11 Gazzani F, et al. WARD: a pneumatic system for body weight relief in gait rehabilitation. Proceedings of 18th Annual International Conference of the IEEE Engineering in Medicine and Biology Society, Amsterdam, NL, paper no. 861, 31/X-3/ XI, 1996

12 Wernig A, Muller S, Nanassy A, Cagol E. Laufband therapy based on 'Rules of Spinal Locomotion' is effective in spinal cord injured persons. Eur J Neurosci 1995; 7: 823-829.

13 Korner-Bitensky $\mathrm{N}$, et al. Motor and functional recovery after stroke: accuracy of physical therapists' predictions. Arch Phys Med Rehabil 1989; 70: $95-99$.

14 Bernardi M, et al. Assessment of energy cost of walking. Project of European Community a-2002 CAMARC. Final report. 1994. pp $87-98$.

15 Bernardi M, et al. Cost of walking and locomotor impairment. $J$ Electromyography Kinesiol, 1999; 9: $149-157$.

16 Zamparo $\mathrm{P}$, et al. The energy cost of level walking in patients with hemiplegia. Scand J Med Sci Sports 1995; 5: 348-352.

17 Sachs L. Applied Statistics. New York, Berlin, Heidelberg, Tokio: Springer-Verlag, 1982

18 Hesse S, Malezic M, Schaffrin A, Mauritz KH. Restoration of gait by combined treadmill training and multichannel electrical stimulation in nonambulatory hemiparetic patients. Scand $J$ Rehabil Med 1995; 27: 199-204.

19 Margaria R. Sulla fisiologia e specialmente sul consumo energetico della marcia e della corsa a varia velocità ed inclinazione del terreno. Atti Acc Naz Lincei 1938; 7: 299-368.

20 Di Prampero PE. The energy cost of human locomotion on land and in water. Int J Sports Med 1986; 7: 55-72.

21 Visentin M, Barbeau H. The effects of parallel bars, body weight support and speed on the modulation of the locomotor pattern of spastic paretic gait. A preliminary communication. Paraplegia 1994; 32: 540 - 553.

22 Basso DM, et al. MASCIS evaluation of open field locomotor scores: effects of experience and teamwork on reliability. Multicenter Animal Spinal Cord Injury Study. J Neurotrauma 1996; 13: $343-359$.

23 Norman KE, Pepin A, Ladouceur M, Barbeau H. A treadmill apparatus and harness support for evaluation and rehabilitation of gait. Arch Phys Med Rehabil 1995; 76: $772-778$.

24 Ide T, Siddiqi NA, Akamaysu N. Expectation for medical and healthcare robotics. JRSJ 1993; 7: 189-200. 